\title{
Desconfianza en el vecino, obstáculo en los planes de comunicación para la cohesión social en sectores rurales de Colombia*
}

\author{
María Cristina Ocampo-Villegas* \\ Miguel Alberto Soto-Ordóñez ${ }^{* * *}$ \\ Recibido: 2017-10-28 • Enviado a pares: 2017-11-06 \\ Aprobado por pares: 2017-12-12 Aceptado: 2018-01-11 \\ DOI: 10.22395/angr.v16n32a4
}

\begin{abstract}
Resumen
El desarrollo de actividades del sector público en Colombia, orientadas a impactar en el nivel de vida de los campesinos, se ve obstaculizado por la falta de credibilidad en las entidades públicas y en el personal externo a la comunidad. No obstante, sorprende, en especial, la falta de confianza en el actuar de los propios miembros del colectivo: los vecinos. Comprender el tema de la desconfianza se convierte en un elemento fundamental dentro de procesos de cohesión social en Colombia, dada la actual coyuntura. El presente artículo analiza el sentimiento de desconfianza entre vecinos de una comunidad rural en el distrito de riego de Monquirá (Boyacá) y pretende aportar elementos para las acciones orientadas a la creación de programas de cohesión social, en escenarios en los que se busque la generación de confianza en comunidades de vecinos. La investigación parte de la pregunta: ¿cuál es el grado de confianza que se tiene en los integrantes de la misma comunidad que asumen roles de liderazgo y de representación frente a las entidades del Estado? Para averiguarlo, se realizó una muestra no probabilística, en la que se aplicó un instrumento no estandarizado basado en los tres niveles de influencia social de Kelman. El trabajo permitió entender el comportamiento de los usuarios del distrito de riego. Se encontró que los individuos temen que los vecinos no cumplirán con los compromisos, y se evidencia una fragmentación de la cohesión social debido a la desconfianza en el accionar del otro.

Palabras clave: comunicación estratégica; desconfianza; influencia social; sector rural; cohesión social; capital social; conformidad; identificación; internalización.
\end{abstract}

Este artículo se desprende de la tesis presentada para optar por el título de Maestría en Comunicación Estratégica de la Universidad de La Sabana (Colombia). Durante el trabajo de investigación para la tesis titulada Propuesta de comunicación estratégica en el distrito de riego de Monquirá, en el departamento de Boyacá, nos encontramos con temas relacionados que vale la pena estudiar con mayor detenimiento. Uno de ellos es el que nos ocupa y que tiene por objeto averiguar las características de la desconfianza en los vecinos y la incidencia de dicho comportamiento en el éxito de los trabajos de intervención en comunicación en las comunidades estudiadas.

*. Doctora en Ciencias de la Comunicación. Centro de Investigación en Comunicación Corporativa y Organizacional (CICCO). Facultad de Comunicación de la Universidad de La Sabana (Colombia). maria. ocampo@unisabana.edu.co. https://orcid.org/0000-0003-4240-0021

... Magíster en Comunicación Estratégica. Comunicador social y periodista. Profesor de la Universidad de La Sabana.miguelso33@gmail.com.https://orcid.org/0000-0002-0085-3156 


\title{
Mistrust in the Neighbor: An Obstacle for the Communication Plans for Social Cohesion in Rural Sectors of Colombia
}

\begin{abstract}
The development of public sector activities in Colombia, aimed at affecting the peasants' standard of living, is hampered by the lack of credibility of public entities and external staff. However, the lack of confidence in the neighbors' actions is specially surprising. Understanding the issue of distrust becomes a fundamental element in social cohesion processes in Colombia, given the current situation. This article analyzes the feeling of distrust among neighbors of a rural community in the irrigation district of Monquirá (Boyacá, Colombia), and intends to contribute elements for actions aimed at creating social cohesion programs, in scenarios in which the trust in neighborhood communities is sought. The investigation starts from the following question: what is the degree of trust in the members of the same community that assume leadership and representation roles in State entities? To find out, a non-probabilistic sample was carried out, in which an unstandardized instrument, based on the three levels of Kelman's social influence, was applied. The study allowed to understand the behavior of the users of the irrigation district. It was found that the individuals fear that the neighbors will not comply with their commitments, and a fragmentation of the social cohesion is evidenced due to the distrust in the actions of the other.
\end{abstract}

Keywords: strategic communication; distrust; social influence; rural sector; social cohesion; social capital; accordance; ID; internalization.

\section{Desconfiança entre vizinho, obstáculo nos planos de comunicação para a coesão social em setores rurais da Colômbia}

\begin{abstract}
Resumo
O desenvolvimento de atividades do setor público na Colômbia, orientadas a impactar no nível de vida dos camponeses, vê-se obstaculizado pela falta de credibilidade nas entidades públicas e no pessoal externo à comunidade. Contudo, surpreende, em especial, a falta de confiança no atuar dos próprios membros do coletivo: os moradores. Compreender o tema da desconfiança se converte em um elemento fundamental em processos de coesão social na Colômbia, tendo em vista a atual conjuntura. Este artigo analisa o sentimento de desconfiança entre os moradores de uma comunidade rural na Distrito de Riego de Monquirá (Boyacá) e pretende contribuir com elementos para as ações orientadas à criação de programas de coesão social em cenários nos quais a geração de confiança é buscada em comunidades de moradores. Esta pesquisa parte da pergunta: qual é o grau de confiança que há nos integrantes da mesma comunidade que assumem papéis de liderança e de representação ante as entidades do Estado? Para respondê-la, foi realizada uma amostra não probabilística, em que foi aplicado um instrumento não padronizado, baseado nos três níveis de influência social de Kelman. Foi constatado que os indivíduos receiam que os vizinhos não cumpram com os compromissos e evidenciada uma fragmentação da coesão social devido à desconfiança no agir do outro.
\end{abstract}

Palavras-chave: comunicação estratégica; desconfiança; influência social; setor rural; coesão social; capital social; conformidade; identificação; internalização. 


\section{Introducción}

Con el fin de propiciar un mayor crecimiento de la economía agrícola en Colombia, el Ministerio de Agricultura diseñó una política de desarrollo rural con enfoque territorial que es ejecutada, en parte, por el Instituto Colombiano de Desarrollo Rural (Incoder).

A partir de este lineamiento, se atiende la provisión de bienes públicos como es la adecuación de tierras, para la administración, conservación y operación de los denominados distritos de riego en diferentes zonas rurales del país (Incoder, 2013). Los distritos de riego buscan reconvertir la productividad de los pequeños productores agropecuarios a partir de transferencia tecnológica y capacitación en esas zonas (Incoder, 2013).

Para lograr dicho objetivo, los distritos de riego se apoyan en procesos de comunicación que toman como base la emisión de mensajes institucionales que se difunden en el campo.

Uno de los mayores problemas que enfrentan las entidades del sector público al momento de relacionarse con sus públicos es la desconfianza que generan. En este sentido, existen trabajos académicos que han abordado el tema de la comunicación de las entidades públicas como los adelantados por Canel (2007, 2009), y Canel y García (2013) sobre gobiernos fiables, o el de Berman (1997) Dealing with Cynical Citizens, ampliamente citado en documentos académicos.

Esta situación no es ajena al Incoder que evidencia una fractura en los procesos de comunicación. El Incoder no se comunica en forma directa con los productores (campesinos o agricultores), pues la información se transmite a través de la Asociación de Usuarios que, si bien son campesinos organizados, sirven de mediadores entre la organización y la totalidad de la comunidad que se desea impactar. Dicha intermediación genera una comunicación deficiente y errónea entre la Asociación y los usuarios productores.

En este panorama de comunicación indirecta, el Incoder identifica como principales consecuencias que los usuarios productores no se relacionan con la Institución, ni tienen interés en involucrarse adecuadamente con los procesos que se desarrollan al nivel productivo. Esto genera una preocupante ausencia de pertenencia y participación del usuario productor con la oferta institucional del Incoder. En un principio, este comportamiento es atribuido a la desconfianza que generan las entidades públicas, a pesar de que el interlocutor directo es una Asociación de Usuarios, es decir, de campesinos organizados e interesados en mejorar las condiciones económicas y de vida de su comunidad.

Por esta razón, se decide indagar por otro aspecto relacionado con el grado de confianza que se tiene en los integrantes de la misma comunidad y la cohesión social que existe entre los miembros, quienes, además, comparten lazos de amistad y parentesco.

En Colombia, un estudio realizado entre 2011 y 2012 analizó la cohesión social de una población de 1200 habitantes de 12 veredas de la zona de Tierralta Córdoba, zona semirural en donde se adelantaban programas de salud pública. El estudio pretendía validar la escala de capital social cognitivo de Wang et al. (2009), aplicada en zonas rurales de China y 
en la cual se validan la confianza y la desconfianza mediante 10 preguntas sobre actitudes de interacción social (Fernández et al., 2014). Los investigadores consideraban la cohesión social como un determinante para los programas de salud. En este análisis, el tema de la desconfianza entre los mismos vecinos fue uno de los elementos que se evidenció.

Se tiene conocimiento también de que la falta de confianza es una debilidad que puede constituirse en un elemento disociador de la comunicación de una comunidad determinada, aun cuando se busca construir capital social. Para Durston (2001), «las actitudes de confianza y las conductas de reciprocidad y cooperación dentro de una comunidad específica» son elementos fundamentales en la construcción de capital social (p. 399).

Asimismo, Arriagada et al., (2003) indican que las redes y actitudes de confianza se construyen a partir del capital social y cultural, así como del carácter de la persona. Esto permite entender que la desconfianza contribuye al deterioro y posible destrucción del tejido social de una comunidad. Cuando se presenta este fenómeno entre los grupos, Flora \& Butler (2003) explican que:

Los grupos no están dispuestos a cooperar entre sí; el conflicto con lo externo se convierte en interno y en el atributo dominante en el plano comunitario. Aunque cada grupo de la comunidad geográfica pueda actuar colectivamente, es difícil organizar y llevar a cabo acciones comunitarias si predomina el conflicto interno (p. 566).

El problema radica en el desconocimiento específico de uno de los tipos principales de capital social y que Islam et al., (2006) definen como estructural. Se refiere a los aspectos observables de la organización social, como son el papel desempeñado por cada individuo, las reglas, los precedentes, los procedimientos y la amplia variedad de redes que contribuyen a la cooperación.

La desconfianza afecta las relaciones horizontales que se presentan entre los grupos de una misma comunidad. Para el caso del distrito de riego Usochicamocha, específicamente en la unidad de Monquirá, en el departamento de Boyacá, objeto de este trabajo, el trato de igual a igual y de comunicación recíproca es afectado por no creer en el actuar y en las decisiones del vecino. No importa que compartan la misma cultura, costumbres, lenguaje, gustos, entre otros. La construcción de participación colectiva se deteriora cuando no se presenta comunicación de confianza.

Bajo este panorama, se puede retomar lo expuesto por Durlauf $\&$ Fafchamps (2004) como conclusiones sobre los efectos sociales del capital social y que evidencian las debilidades de la comunidad objeto de estudio:

(a) El capital social genera externalidades positivas para los miembros de un grupo, (b) estas externalidades se obtienen gracias a la confianza compartida, normas y valores como también por sus efectos sobre las expectativas y el comportamiento, y (c) la confianza compartida, normas y valores se derivan de las modalidades informales de las organizaciones basadas en la red social y de asociación (p. 2). 
La confianza, por lo tanto, es una condición que se logra a partir de las relaciones que se establecen con las otras personas y que está altamente implicada por la comprensión mutua y la simetría en las relaciones (Gruning y Hont, 1999). Otros autores, como Lewicki $\varepsilon$ Benedict (1996), identifican tres perspectivas diferentes para analizar la confianza: la perspectiva de la disposición o de la personalidad, que se centra en las diferencias de personalidad en los individuos; la perspectiva sociológica y económica, que se centra en la confianza como un fenómeno institucional, y la perspectiva de la psicología social, que se centra en las transacciones interpersonales entre los individuos. Miller y Mitamura (2003) y Couch y Jones (1997) se refieren al concepto de confianza social como confianza generalizada hacia todas las personas; esta sería una expectativa sobre la buena voluntad que tendrían los seres humanos.

La confianza social, abordada por Kramer (1999) como disposicional, es definida por este autor como la concepción que se tiene sobre cómo es la naturaleza humana. Es decir, alguien con alta confianza social o generalizada cree, por ejemplo, que la mayoría de las personas tienen buenas intenciones, son honestas y respetuosas.

Dentro de este marco, el presente estudio permitió identificar la existencia de un fenómeno de desconfianza por parte de los campesinos usuarios del Distrito hacia sus vecinos, en especial cuando se plantea la posibilidad de cumplir, de manera colectiva, las decisiones o lineamientos dispuestos por el Incoder.

Durston (2002) explica que los mismos precursores de la confianza y la cooperación - parentesco, identidad étnica, prestigio del servicio comunitario- pueden dar lugar a graves conflictos entre individuos y entre facciones. La competencia y la rivalidad entre hermanos es un fenómeno universal; la competencia por ser el máximo y único líder de una comunidad lleva esta rivalidad al plano social y desata confrontaciones aun entre grupos unidos por el parentesco y la amistad.

\section{Método}

El presente documento surge de una investigación de mayor alcance, la cual correspondió a un proyecto que contempló una propuesta de comunicación estratégica para el distrito de riego Usochicamocha, específicamente en la unidad de Monquirá, en el departamento de Boyacá-Colombia.

La unidad de Monquirá del distrito de riego Usochicamocha se ubica a tres horas de Bogotá D. C. Es una región de clima frío, entre los 3 y 15 grados Celsius. Es considerada una zona estratégica para el país por la producción de alimentos como papa, lechuga, cebolla, zanahoria, fríjol, alverja, mora, tomate de árbol, entre otros. Además, por su ubicación geográfica, es un corredor terrestre importante al conectar el centro del país con los Llanos Orientales.

El distrito Usochicamocha pretende impactar, con su oferta de servicios, a un grupo de 720 campesinos usuarios distribuidos en cuatro sectores de la unidad Monquirá (El 
Cerrito, Caparral Centro, Río Chiquito y Jardines). Dichos campesinos viven por debajo de la línea de pobreza. No obstante, su nivel educativo es Primaria Básica y bachillerato académico. Por ende, saben leer y escribir. Además, son poseedores de una extensión de 1 a 2 hectáreas de tierra, es decir, cerca de 5000 metros cuadrados, lo que los califica como minifundistas.

En el instrumento diseñado para evaluar las condiciones de percepción sobre los mensajes emitidos por la entidad, se contempló una variable relacionada con la confianza que los usuarios tenían en los otros miembros de la misma comunidad, es decir, la confianza en el comportamiento de los vecinos.

La metodología empleada para intervenir a la población objetivo fue la de talleres experimentales propuestos por Kelman (1958) para analizar los cambios de comportamientos de las personas a través de los niveles de la influencia social (identificación, conformidad e internalización). Este autor afirma que "al saber algo sobre la naturaleza y la profundidad de los cambios, podremos realizar predicciones significativas sobre la forma en que las actitudes cambian. Esto se reflejará en acciones y reacciones a cada evento" (p. 51).

Para obtener los resultados, fue necesario comunicar el mensaje del Instituto a través de cuatro interlocutores diferentes, lo que contribuyó a entender el cambio de comportamiento hacia la desconfianza cuando el interlocutor era un vecino.

\section{Aplicación del método Kelman al caso de Usochicamocha}

Se tuvieron en cuenta los tres niveles de Kelman (1958) para entender los cambios de actitud de la comunidad de usuarios productores de la unidad de riego "Monquirá" del distrito de riego Usochicamocha. Los niveles de Kelman se definen de la siguiente manera:

La conformidad: ocurre cuando una persona acepta la influencia porque espera alcanzar una reacción favorable de otro individuo o grupo. La conformidad se debe a los efectos sociales de aceptar la influencia (Kelman, 1958, p. 53).

La identificación: se presenta cuando una persona acepta la influencia porque quiere establecer o mantener una satisfacción definida al relacionarse con otra persona o grupo. Se identifica cuando se opta por tomar el rol de otra persona, quien adopta el comportamiento porque lo asocia con una relación deseada. La identificación se debe a un acto de ser parte o conformar un grupo (Kelman, 1958, p. 53).

La internalización: se evidencia cuando una persona acepta la influencia porque el contenido del comportamiento (ideas y acciones de las cuales se compone) es provechoso o resulta satisfactorio. Adopta el comportamiento porque es congruente con su sistema de valores. La internalización se debe al contenido del nuevo comportamiento (Kelman, 1958, p. 53).

Estos niveles, aplicados a los usuarios productores, ayudaron a identificar cuáles son las actitudes y comportamientos que tiene la población de la unidad de riego "Monquirá" frente al trabajo que ha venido desarrollando el Incoder a través del distrito de riego Usochicamocha. 
Con ello, se identificaron las variables centrales con las cuales se explican las fallas de comunicación que impiden la transformación social y cultural de la comunidad para que sea más cercana al Instituto y goce de mayores beneficios.

\section{Desarrollo de la investigación}

Para la aplicación de los talleres experimentales se plantean dos fases:

Primera fase, la comunicación: el Incoder considera como uno de los principales compromisos de los usuarios de Usochicamocha, la inscripción en el distrito de riego. Basados en esta indicación de la entidad se grabó en audio, pero con cuatro interlocutores diferentes: un directivo de la entidad, un vocero o líder de la comunidad intervenida, un experto en el tema de la decisión y una persona externa y ajena tanto al grupo como a la entidad.

Los usuarios fueron convocados en forma voluntaria para participar de los grupos focales o talleres. Se organizaron cuatro grupos, uno de los cuales cumple la función de grupo de control. Los tres grupos restantes responden a las características que propone Kelman para conformidad, identificación e internalización. A cada uno de los grupos se les expuso a uno de los audios.

Segunda fase, sondeo: la segunda fase consiste en la aplicación de cuestionarios de 18 ítems, a los cuatro grupos focales diferentes (uno por cada nivel de la influencia social y otro de control-comparación). La escala de calificación de cada pregunta correspondía a 1 (total descuerdo) y 5 (total acuerdo), de acuerdo con Likert (1932).

Asimismo, la aplicación de los cuestionarios se realizó bajo condiciones de vigilancia (solicitud de datos personales y supervisión de quien emite el mensaje de la comunicación) y de relevancia (retención de la información comunicada).

Se tradujo la versión original del instrumento de Kelman (1958) a español y, posteriormente, se modificaron algunos lineamientos de su aplicación debido a que la participación fue voluntaria. La medición se llevó a cabo también en dos etapas:

En la primera etapa, los datos se midieron en forma individual. Se procedió a la tabulación tomando en cuenta los totales de cada persona una vez se respondían los 18 puntos de la encuesta. Con ello, el rango de calificación sobre el total de preguntas fue 18 (completo desacuerdo) o 90 (total acuerdo). Para el análisis de los datos, se dividieron los rangos de respuesta en cuartiles de la siguiente manera:

- $\quad$ Primer cuartil $=$ entre 18 y $22.5=$ Completo desacuerdo.

- Segundo cuartil $=$ entre 22.6 y 45. Poco acuerdo.

- $\quad$ Tercer cuartil $=$ entre 45.1 y 67.5. Acuerdo medio.

- $\quad$ Cuarto cuartil $=$ entre 67.6 y $90=$ Total acuerdo. 
La segunda medición se efectuó en forma grupal, por pregunta. Se obtuvo la media de las respuestas por pregunta y se catalogó según la siguiente escala:

- $\quad$ Rango entre 1 y 2: Ningún acuerdo

- $\quad$ Rango entre 2.1 y 3: Poco acuerdo

- $\quad$ Rango entre 3.1 y 4: Acuerdo medio

- $\quad$ Rango entre 4.1 y 5: Total acuerdo

\section{Análisis y resultados}

Según los niveles de conformidad, identificación e internalización, se aislaron las preguntas que hacían referencia a la confianza en los vecinos o en la comunidad para cumplir con las indicaciones del Incoder. Igualmente, se aislaron las preguntas referentes al comportamiento individual del usuario frente al cumplimiento de las indicaciones dadas por la entidad. Como se puede apreciar en la tabla 1, las variables con comportamiento más crítico se refieren a la percepción que tienen los usuarios sobre el comportamiento de sus pares, es decir, de sus vecinos y de la comunidad en general.

Tabla 1. Variables críticas en los cuestionarios de conformidad, identificación e internalización

\begin{tabular}{|c|l|l|c|}
\hline \multicolumn{1}{|c|}{ Nivel } & \multicolumn{1}{|c|}{ Pregunta } & \multicolumn{1}{|c|}{ Variable } & Promedio \\
\hline Conformidad & $\begin{array}{l}\text { ¿Piensa usted que sus vecinos están dispues- } \\
\text { tos a actualizar o inscribirse en el Registro } \\
\text { General de Usuarios del Distrito? }\end{array}$ & $\begin{array}{l}\text { Disposición de los ve- } \\
\text { cinos a inscribirse en el } \\
\text { Registro }\end{array}$ & 2,8 \\
\hline Conformidad & $\begin{array}{l}\text { ¿Cree usted que sus vecinos cumplirán la } \\
\text { decisión tomada por el Incoder? }\end{array}$ & $\begin{array}{l}\text { Cumplimiento de los ve- } \\
\text { cinos con la decisión del } \\
\text { Incoder }\end{array}$ & 2,6 \\
\hline \multirow{2}{*}{ Identificación } & $\begin{array}{l}\text { ¿Cree usted que sus vecinos seguirán las } \\
\text { recomendaciones del delegado de la Unidad } \\
\text { Monquirá ante la Junta Directiva? }\end{array}$ & $\begin{array}{l}\text { Seguimiento de los veci- } \\
\text { nos a las recomendacio- } \\
\text { nes del delegado }\end{array}$ & 2,5 \\
\hline Identificación & $\begin{array}{l}\text { ¿Piensa usted que la comunidad seguirá } \\
\text { al pie de la letra las indicaciones que dio a } \\
\text { conocer el delegado? }\end{array}$ & $\begin{array}{l}\text { Seguimiento de los veci- } \\
\text { nos a las recomendacio- } \\
\text { nes del delegado }\end{array}$ & 2,9 \\
\hline Internalización & $\begin{array}{l}\text { Sin escuchar la intervención del experto, } \\
\text { ¿́se habría inscrito o actualizado el Registro } \\
\text { General de Usuarios del Distrito? }\end{array}$ & Inscripción al registro & 2,8 \\
\hline
\end{tabular}

Fuente: elaboración propia

\section{Grupo focal 1}

Nivel de conformidad: se reprodujo el audio de la Directora Técnica de Administración de Distritos del Incoder. Es decir, esta persona representaba un cargo directivo y de toma de decisiones del Instituto frente a los usuarios. 
El promedio para la pregunta ¿piensa usted que sus vecinos están dispuestos a actualizar o inscribirse en el Registro General de Usuarios del Distrito? fue de 2,8. Dicho resultado contrasta con el promedio obtenido en la pregunta ¿piensa usted que es una obligación para los usuarios productores inscribirse en el Registro General de Usuarios del Distrito? cuyo resultado fue de 4,2.

Asimismo, la respuesta de los usuarios a la pregunta ċla decisión del Incoder, basada en la Ley 41, lo presiona a cumplir con el Registro General de Usuarios de Distrito? fue en promedio de 3.5 .

Esta posición se acentúa al observar la respuesta a la pregunta ¿̇e siente obligado a acercarse a la Asociación de Usuarios del Distrito a diligenciar el Registro General de Usuarios?, que fue calificada en promedio con 3,1, es decir, acuerdo medio.

Para los investigadores, esta información indica que las decisiones del Incoder, a pesar de formularse con un amparo normativo, son de poco acogimiento por parte de los usuarios. Por lo anterior, se puede inferir que los usuarios consideran una «obligación» inscribirse en el Registro, pero desconfían de que los vecinos estén dispuestos a realizar dicho registro. Y ellos mismos no se sienten del todo obligados a cumplir con la normativa.

Los resultados también demuestran que existe desconfianza respecto a la actitud que tome la comunidad frente al cumplimiento de una obligación impuesta por el Incoder. Lo anterior se desprende del promedio de respuestas a la pregunta $N$. $^{\circ}$, ¿̇cree usted que sus vecinos cumplirán la decisión tomada por el Incoder?, que se ubica en 2,6.

Nivel de identificación: Para el taller experimental 2, se reprodujo el audio del líder o vocero de los usuarios frente a la Junta Directiva de Usochicamocha. Es decir, una persona de la comunidad que representa las opiniones y posiciones de esta frente a las directivas del distrito.

Bajo esta perspectiva, el promedio de los usuarios se encuentra en acuerdo medio (calificación de 3,2) frente a la pregunta ćconsidera que las opiniones del delegado de la unidad Monquirá ante la Junta Directiva de Usochicamocha son provechosas para la comunidad?

Aunque se tiene esta conducta, los encuestados se encuentran en total acuerdo (promedio 4,0) frente a la variable «familiaridad con lo expuesto» de la pregunta $\mathrm{N}^{\circ}{ }^{4}$, lo que indica que los usuarios, a pesar de familiarizarse con la información transmitida por su líder de manera individual, tienen un acuerdo medio frente a lo válidas o provechosas que resultan las opiniones del vocero para el resto de la comunidad. En otras palabras, como actores individuales los usuarios se comportan en total acuerdo, pero su actitud cambia a desconfianza, al involucrarse con la comunidad.

Asimismo, los usuarios califican con un promedio de 2,5 la pregunta ċcree usted que sus vecinos seguirán las recomendaciones del delegado de la Unidad Monquirá ante la Junta Directiva? El análisis muestra que los usuarios perciben que sus vecinos poco 
siguen las recomendaciones de su líder para cumplir con las indicaciones del Incoder. Se plantea entonces un problema de confianza que parte del usuario, de manera individual, frente al seguimiento de unas recomendaciones que fueron promovidas por la misma comunidad a través de su vocero.

Los resultados también llamaron la atención frente a la pregunta custed respaldaría las opiniones expuestas por el delegado de la unidad de Monquirá? El promedio se ubicó en acuerdo medio $(3,2)$. Sin embargo, al plantear la variable «seguimiento de los vecinos a las recomendaciones del delegado» el promedio arrojado fue de poco acuerdo $(2,9)$. Esto corrobora la posición de los encuestados de desconfianza y poca aprobación hacia su propio líder. Esto, sin importar que él recopile opiniones de sus vecinos sobre las medidas y recomendaciones por parte del Incoder.

Se evidencia un comportamiento recurrente. Los usuarios tienen poco acuerdo en actuar de manera colectiva frente al cumplimiento de una indicación institucional, lo cual radica en la desconfianza. Además, los resultados muestran que el individuo poco confía en que su vecino (par o similar) cumpliría las decisiones impuestas.

Nivel de internalización: en el taller experimental 3, antes de aplicar los sondeos, se reprodujo el audio del experto en adecuación de tierras. Es decir, un especialista en el tema, con todo el reconocimiento en el sector agrícola por su recorrido en distritos de riego.

Bajo esta perspectiva, los usuarios manifestaron, en promedio, un acuerdo medio $(3,5)$ en la variable «promover la recomendación» (del Incoder) en la pregunta ¿cree usted que sus vecinos promoverían la actualización o inscripción en el Registro General de Usuario del Distrito?

Además, los participantes del taller calificaron con un promedio de 2,8 (poco acuerdo) la variable «inscripción del registro», de la pregunta: sin escuchar la intervención del experto, ¿̇se habría inscrito o actualizado el Registro General de Usuarios del Distrito? Se resalta el enfoque de la pregunta en realizar el procedimiento antes de escuchar al experto.

Es curioso encontrar que la actitud de los sondeos es de poco acuerdo a inscribirse en el Registro Único de Usuario sin haber oído al experto. Es decir, el promedio de los asistentes valida los argumentos del especialista, lo que incentiva a cambiar su posición y a acceder a lo solicitado por el Instituto.

Lo anterior se valida con el siguiente hallazgo: los usuarios tienen un acuerdo medio $(3,8)$ frente a la pregunta custed cree que los demás usuarios se acogerán al Registro General de Usuarios del Distrito, después de que el experto comunicara los beneficios?

Se puede concluir que realmente hay un nivel de internalización. El experto realmente influye en el cambio de posición de los usuarios frente al cumplimiento de las recomendaciones del Incoder. Es claro que sin tener dichas declaraciones, el promedio de los usuarios estaría poco de acuerdo en tomar la iniciativa de actualizar el Registro Único de Usuarios. 


\section{Discusión y conclusiones}

El estudio abre una línea de base para la elaboración de futuras investigaciones orientadas a conocer el relacionamiento entre entidades públicas y población rural. Igualmente, se puede ahondar en la investigación del fenómeno de la desconfianza entre las comunidades rurales. Este tipo de investigaciones se constituyen en factores indispensables para la articulación de las acciones gubernamentales que apoyen procesos de desarrollo social en comunidades rurales con diversidad de actores, como los que actualmente se adelantan en la etapa del posconflicto.

El estudio evidenció una fuerte inclinación de la población rural por cumplir con las recomendaciones de una entidad estatal, de manera individual. Hay alta conformidad, identificación e internalización, sin importar el interlocutor que transmita el mensaje.

Sin embargo, el comportamiento de los usuarios cambia cuando se trasladan al escenario de si sus vecinos o la comunidad en general cumplirían las indicaciones. Hay poca conformidad e identificación en este panorama. Los individuos, cuando piensan en el accionar de los demás, en el colectivo, temen que los otros no cumplirán las indicaciones, lo que evidencia una fragmentación de la cohesión social debido a la desconfianza en el accionar del otro.

Como lo ponen de manifiesto Durlauf $\&$ Fafchamps (2004) los efectos sociales de la desconfianza evidenciada se ven reflejados en las externalidades, en este caso negativas, en la unidad de Monquirá. Los problemas encontrados de falta de relacionamiento con la institución, falta de interés en involucrarse en los procesos y falta de sentido de pertenencia son consecuencia de dicha percepción. A la vez, dicho comportamiento pone en riesgo la efectividad de las comunicaciones de la Institución.

Lo anterior se aclara con los resultados de las variables de internalización. Los usuarios cambian sus comportamientos cuando una persona externa les explica y motiva a cumplir las directrices de la Institución. Sin escuchar a un experto, su comportamiento es poco probable que cambie. De ahí la importancia de incorporar expertos externos dentro de los procesos de cohesión social que se avecinan y en los cuales es muy probable que se presente un alto componente de desconfianza entre los miembros de las comunidades receptoras de excombatientes.

Teniendo en cuenta lo planteado por Flora \& Butler (2003) frente a la disponibilidad de cooperar al nivel comunitario, cuando existe desconfianza, y lo expresado por Islam et al., (2006) quienes atribuyen la desconfianza al desconocimiento de las reglas, precedentes y procedimientos que conforman las redes de cooperación, se plantean las siguientes recomendaciones.

1. Para reducir la desconfianza en el ámbito rural y comunicativo, se debe estructurar una estrategia pedagógica que impulse el conocimiento de los derechos, deberes, oferta institucional y novedades del distrito de riego con la población, a través de espacios 
de relacionamiento horizontal (trato de igual a igual) que aumenten el protagonismo de los usuarios productores.

2. Para lograr la eficacia de la comunicación, esta debe ser gestionada a través de la figura de un agente externo (experto en temas agrícolas) que permita transmitir la comunicación y mejorar la confianza entre la población.

Dentro de las estrategias sugeridas a la Institución, se utilizan mecanismos de mediación y de socialización, ya que, como lo advierten Abellán y Mayugo (2008), son «dos aspectos complementarios que aúnan los campos de la educación y la comunicación para contribuir a una reestructuración de las dimensiones de convivencia, cohesión social y transformación» (p. 130).

3. Los investigadores también proponen, tanto al Incoder como a entidades gubernamentales, la creación de redes de aprendizaje informales que, como lo plantean Sloep y Berlanga (2011), contribuyen al intercambio de experiencias y conocimientos, apoyan el trabajo colaborativo, crean grupos de trabajo y permiten ofrecer y recibir apoyo de otros usuarios.

4. Asimismo, se recomienda capacitar en liderazgo y comunicación efectiva a los usuarios productores que actualmente participan de la oferta institucional del Incoder y, en sí, del Gobierno Nacional (voceros activos de la buena imagen y oferta institucional) para que sensibilicen a la comunidad. En pocas palabras, es empoderar a los participantes activos para motivar y ganar credibilidad del trabajo colectivo.

5. Mantener las bases de la idiosincrasia de la comunidad, basadas en trabajo de cooperación y de involucramiento de igual a igual, permitirá reconocer que el vecino también trabaja en pro del bien común y no de sus intereses particulares. Por ende, aumentar su sentido de pertenencia con actividades de experiencia sensorial y construyendo un discurso que se identifique con las emociones de los campesinos permitirá incrementar el capital social de manera sostenible.

\section{Referencias}

Abellán, G. y Mayugo, C. (2008). La dimensión comunitaria de la educación en comunicación. Comunicar, 16(31), 129-136.

Arriagada, I., Atria, R., Robison, L., Siles, M. y Whiteford, S. (2003). Capital social y reducción de la pobreza en América Latina y el Caribe: en busca de un nuevo paradigma. Santiago de Chile: Cepal Editores.

Berman, E. (1997). Dealing with cynical citizens. Public Administration Review, 57(2), 105-1 12.

Canel, M. J. (2007). La comunicación de las instituciones públicas. Madrid: Tecnos.

Canel, M. J. (2009). El impacto de los sucesos imprevistos en la imagen pública de los gobiernos españoles. Análisis, (38), 219-236.

Canel, M. J. y García, A. (2013). Comunicar gobiernos fiables. Análisis de la confianza como valor intangible del Gobierno de España. Revista ZER, 18(34), 29-48. 
Couch, L. y Jones, W. (1997). Measuring Levels of Trust. Journal of Research in Personality, 31, 319-336.

Davis, F., Bagozzi, R. y Warshaw, P. (1989). User acceptance of computer technology: a comparison of two theoretical models. Management Science, 35, 982-1003.

Durlauf, S. y Fafchamps, M. (2004). Social capital Cambridge: National Bureau of Economic Research En: Philippe Aghion y Steven Durlauf (ed.) Manual de crecimiento económico (pp. 1639-1699) Cambridge, Massachusets: Elsevier.

Durston, J. (2002). El capital social campesino en la gestión del desarrollo rural. Díadas, equipos, puentes y escaleras. Santiago de Chile, Chile: Publicación de las Naciones Unidas.

Durston, J. (2001). Parte del problema, parte de la solución: el capital social en la superación de la pobreza en América Latina y el Caribe. En Libros de la Cepal (Ed.), Capital social y reducción de la pobreza en América Latina y el Caribe: en busca de un nuevo paradigma (pp.147-202). Santiago de Chile, Chile: Publicación de las Naciones Unidas.

Fernández, J., Pinzón, C. y Moreno, J. (2014). Capital social en áreas rurales: adaptación al español y validación factorial de una escala. Ciencia y Salud Colectiva, 19(7), 2207-2214.

Flora, J. y Butler, C. (2003). Desarrollo comunitario en las zonas rurales de los Andes. En I. Arriagada, R. Atria,L. Robison, M. Siles y S. Whiteford (Eds.), Capital social y reducción de la pobreza en América Latina y el Caribe: en busca de un nuevo paradigma (pp. 555-578). Santiago de Chile: Cepal Editores.

Grunig, J. E., y Hon, L. (1999). Guidelines for measuring relationships in Public Relations. Florida, Estados Unidos: The Institute for Public Relations.

Islam, M. K., Merlo, J., Kawachi, I., Lindstrom, M. y Gerdtham, U. G. (2006). Social capital and health: does egalitarianism matter? A literature review. International Journal for Equity in Health. Recuperado de https://equityhealthj.biomedcentral.com/articles/10.1186/1475-9276-5-3

Sloep, P. y Berlanga, A. (2011). Redes de aprendizaje, aprendizaje en red. Comunicar, 19 (37), 55-64.

Wang, H., Schlesinger, M. y Hsiao, W. C. (2009). The flip-side of social capital: the distinctive influences of trust and mistrust on health in rural China. Social Sciences and Medicine, 68(1), 133-142. 